\title{
Who Will Benefit? Using Radiomics to Predict Response to Oxaliplatin-Based Chemotherapy in Patients with Colorectal Liver Metastases
}

\author{
Daniel Brock Hewitt, MD, MPH, MS, Timothy M. Pawlik, MD, MPH, PhD, and Jordan M. Cloyd, MD $\mathbb{D}$ \\ Department of Surgery, Division of Surgical Oncology, The Ohio State University Wexner Medical Center, Columbus, OH
}

The liver is the most common site of metastatic disease among patients with colorectal cancer (CRC), with over one-half developing hepatic metastases at some point during their disease course. ${ }^{1}$ Fortunately, improved multidisciplinary cancer care has nearly doubled the 5-year overall survival (OS) rate of patients with colorectal liver metastases (CRLM), reaching 60\% in some patient populations. ${ }^{2,3}$ Improvements in long-term outcomes are due to both advances in surgical approach and the development of more effective systemic agents. ${ }^{4}$ In fact, more potent systemic chemotherapy has dramatically shifted the paradigm of care for patients with CRLM, expanding the indications for resection by downsizing CRLM, as well as treating micrometastatic disease more effectively. ${ }^{5}$

Among patients with unresectable CRLM, chemotherapy is first-line treatment; in turn, about one-third of patients with initially unresectable disease will experience sufficient downsizing to allow for subsequent resection. ${ }^{6,7}$ For patients with resectable CRLM, the use of neoadjuvant chemotherapy may also hold several benefits. Specifically, neoadjuvant chemotherapy may provide important prognostic information, thereby aiding patient selection. In addition, preoperative chemotherapy can facilitate a parenchymal-sparing approach by downsizing metastases. While the optimal chemotherapy regimen to treat patients with CRLM depends on several factors, including previous chemotherapy exposure and the presence of certain

(C) Society of Surgical Oncology 2021

First Received: 29 December 2020

Accepted: 30 December 2020;

Published Online: 21 January 2021

J. M. Cloyd, MD

e-mail: jordan.cloyd@osumc.edu molecular markers, first-line chemotherapy typically includes oxaliplatin or irinotecan with 5-fluorouracil (e.g. FOLFOX or FOLFIRI), occasionally in combination (e.g. FOLFOXIRI), or with a biologic agent.

Despite an interest in promoting personalized approaches to cancer therapy, few biomarkers are currently available to guide individualized decision making for patients with advanced CRC. While Kras mutations and microsatellite instability status are important molecular markers for predicting response to epidermal growth factor and immune checkpoint inhibitors, respectively, there are currently no validated biomarkers to predict response to first line oxaliplatin- or irinotecan-based chemotherapy. Such tools could have important implications relative to the management of patients with CRLM, particularly for patients predicted to be non-responders. Specifically, patients with unresectable disease could be prioritized for alternative non-oxaliplatin-based therapies, hepatic artery infusion, targeted agents guided by next-generation tumor sequencing, or clinical trials. In addition, patients with resectable disease might be considered for upfront surgery.

In the current article, Nakanishi et al. reported on the use of radiomics texture analysis to assist in the prediction of CRLM response to oxaliplatin chemotherapy. ${ }^{8}$ Radiomics uses data characterization algorithms to generate a comprehensive quantification of tumor phenotypes (e.g. tumor texture, intensity, shape) from standard radiographic images. Nakanishi and colleagues analyzed 126 CRLMs from 42 patients (training cohort $[n=94]$ vs. validation cohort $[n=32])$ to test a radiomic-generated score to predict CRLM response to first-line oxaliplatin-based chemotherapy. The authors utilized a least absolute shrinkage and selection operator (LASSO) technique to correlate the radiomics score with response to chemotherapy (defined as complete or partial response by Response Evaluation 
Criteria in Solid Tumors [RECIST] 1.1). In the training cohort, among 1038 candidate radiomic features extracted from each tumor region of interest, 11 features were selected to create the radiomics score. On univariate analysis, the radiomics score was associated with a good response to oxaliplatin-based chemotherapy $(p<0.0001)$, with an AUC of 0.85. This association was maintained in the validation cohort and on multivariable analysis. While the radiomics score was also associated with a decrease in CEA levels related to chemotherapy, the score was not predictive of progression-free survival or OS.

The study provides data to support the feasibility of a radiomics texture-based signature to predict response of CRLM to chemotherapy. While the data are intriguing, several limitations of the study need to be considered. First, the median number of cycles of oxaliplatin-based chemotherapy prior to radiographic assessment was 5.5, yet the interquartile range spanned 4-10 cycles. Thus, the timing of radiographic evaluation relative to the number of cycles may have influenced the results. Second, patients with multiple CRLM lesions can often have a 'mixed response'. Therefore, classifying patients as 'responders' versus 'non-responders' may be an oversimplification. Third, while RECIST is a commonly used tool to measure radiographic response to chemotherapy, changes in CRLM tumor diameter do not always correlate with response to chemotherapy. Measuring morphologic response is an alternative approach which has been associated with histologic response and survival. ${ }^{9}$ Fourth, response was assessed using computed tomography (CT), which may be inferior to magnetic resonance imaging (MRI) in assessing response to chemotherapy among subsets of patients (e.g. steatosis; disappearing liver metastases). ${ }^{10,11}$ Fifth, while scans were reviewed by oncologists, other investigators have demonstrated the implementation of fully automated machine learning algorithms to assess CT scans and generate risk-based scores to predict outcomes. ${ }^{12}$ Finally, the study suffered from a small sample size, as evidenced by the high point estimates and very large 95\% confidence intervals. As such, a larger external cohort will be necessary to validate the utility of the radiomic tool to serve as a predictive biomarker.

The current study by Nakanishi et al. reflects a growing interest in the use of radiomics to personalize treatment for patients with CRC. ${ }^{13}$ For example, recent studies have highlighted the potential for radiomic-based approaches to predict the histological growth pattern of CRLMs, tumor response to therapy, the development of metachronous CRLMs, and even OS. ${ }^{13-15}$ The radiomics score derived by Nakanishi et al. ${ }^{13}$ confirms the utility of skewness as a biomarker for chemotherapy response, which has been observed in previous studies, and also expands the field of candidate radiologic features that could be evaluated in future studies. While radiomics holds promise, this tool will need to be integrated into other approaches aimed at advancing precision oncology for patients with metastatic CRC. For example, next-generation tumor sequencing and histological analysis can identify predictive biomarkers that enable the selection of targeted therapies (e.g. KRAS or BRAF mutations, mismatch repair deficiency). In addition to radiomics and genomics, active investigation in the areas of proteomics, ${ }^{16}$ circulating tumor $\mathrm{DNA}^{17}$ the microbiome, ${ }^{18}$ and patient-derived tumor organoids ${ }^{19}$ hold promise for major advances in personalized approaches to treating CRLM in the near future.

In summary, the contemporary management of CRLM continues to rapidly evolve as advances in surgical approaches, locoregional treatments, and systemic therapies occur. Innovative approaches to predict patient response and guide the optimal selection of an increasing array of treatment options are thus urgently needed. The study by Nakanishi et al. highlights the potential for a radiomics approach as well as some challenges inherent in designing and validating image-based biomarkers. Future efforts will require innovative interdisciplinary approaches to solve these problems as well as international multidisciplinary teams to prospectively validate these novel biomarkers as advances in precision oncology aim to improve the care of patients with CRLM.

DISCLOSURES Daniel Brock Hewitt, Timothy M. Pawlik, and Jordan M. Cloyd declare no conflicts of interest.

\section{REFERENCES}

1. Arnold M, Sierra MS, Laversanne M, et al. Global patterns and trends in colorectal cancer incidence and mortality. Gut. 2017;66(4):683-91.

2. Fernandez FG, Drebin JA, Linehan DC, et al. Five-year survival after resection of hepatic metastases from colorectal cancer in patients screened by positron emission tomography with F-18 fluorodeoxyglucose (FDG-PET). Ann Surg. 2004;240(3):438-47 (discussion 447-50).

3. Chen EY, Mayo SC, Sutton T, et al. Effect of time to surgery of colorectal liver metastases on survival. J Gastrointest Cancer. Epub 22 Feb 2020.

4. Creasy JM, Sadot E, Koerkamp BG, et al. Actual 10-year survival after hepatic resection of colorectal liver metastases: what factors preclude cure? Surgery. 2018;163(6):1238-44.

5. Mayo SC, Heckman JE, Shore AD, et al. Shifting trends in liverdirected management of patients with colorectal liver metastasis: a population-based analysis. Surgery. 2011;150(2):204-16.

6. Adam R, Wicherts DA, de Haas RJ, et al. Patients with initially unresectable colorectal liver metastases: is there a possibility of cure? J Clin Oncol. 2009;27(11):1829-35.

7. Masi G, Loupakis F, Pollina L, et al. Long-term outcome of initially unresectable metastatic colorectal cancer patients treated with 5-fluorouracil/leucovorin, oxaliplatin, and irinotecan (FOLFOXIRI) followed by radical surgery of metastases. Ann Surg. 2009;249(3):420-5. 
8. Nakanishi R, Oki E, Hasuda H, et al. Radiomics texture analysis for the identification of colorectal liver metastases sensitive to first-line oxaliplatin-based chemotherapy. Ann Surg Oncol. 2021. https://doi.org/10.1245/s10434-020-09581-5.

9. Chun YS, Vauthey JN, Boonsirikamchai P, et al. Association of computed tomography morphologic criteria with pathologic response and survival in patients treated with bevacizumab for colorectal liver metastases. JAMA. 2009;302(21):2338-44.

10. Tsilimigras DI, Ntanasis-Stathopoulos I, Paredes AZ, et al. Disappearing liver metastases: a systematic review of the current evidence. Surg Oncol. 2019;29:7-13.

11. Park MJ, Hong N, Han K, et al. Use of imaging to predict complete response of colorectal liver metastases after chemotherapy: MR imaging versus CT imaging. Radiology. 2017;284(2):423-31.

12. Stemmer A, Shadmi R, Bregman-Amitai O, et al. Using machine learning algorithms to review computed tomography scans and assess risk for cardiovascular disease: retrospective analysis from the National Lung Screening Trial (NLST). PLOS ONE. 2020;15(8):e0236021.

13. Fiz F, Viganò L, Gennaro N, et al. Radiomics of liver metastases: a systematic review. Cancers (Basel). 2020;12(10):2881.

14. Ahn SJ, Kim JH, Park SJ, et al. Prediction of the therapeutic response after FOLFOX and FOLFIRI treatment for patients with liver metastasis from colorectal cancer using computerized CT texture analysis. Eur J Radiol. 2016;85(10):1867-74.

15. Dercle L, Lu L, Schwartz LH, et al. Radiomics response signature for identification of metastatic colorectal cancer sensitive to therapies targeting EGFR pathway. $J$ Natl Cancer Inst. 2020;112(9):902-12.

16. Chauvin A, Boisvert FM. Clinical proteomics in colorectal cancer, a promising tool for improving personalised medicine. Proteomes. 2018;6(4):49.

17. Dasari A, Morris VK, Allegra CJ, et al. ctDNA applications and integration in colorectal cancer: an NCI Colon and Rectal-Anal Task Forces whitepaper. Nat Rev Clin Oncol. 2020;17(12):757-70.

18. Sánchez-Alcoholado L, Ramos-Molina B, Otero A, et al. The role of the gut microbiome in colorectal cancer development and therapy response. Cancers (Basel). 2020;12(6):1406.

19. Ooft SN, Weeber F, Dijkstra KK, et al. Patient-derived organoids can predict response to chemotherapy in metastatic colorectal cancer patients. Sci Transl Med. 2019;11(513):eaay2574.

Publisher's Note Springer Nature remains neutral with regard to jurisdictional claims in published maps and institutional affiliations. 